\title{
THE SPANS OF FIVE STAR-LIKE SIMPLE CLOSED CURVES
}

\author{
Thelma West \\ University of Louisiana at Lafayette, USA
}

\begin{abstract}
Let $X$ be a continuum, that is a compact, connected, nonempty metric space. The span of $X$ is the least upper bound of the set of real numbers $r$ which satisfy the following conditions: there exists a continuum, $C$, contained in $X \times X$ such that $d(x, y)$ is larger than or equal to $r$ for all $(x, y)$ in $C$ and $p_{1}(C)=p_{2}(C)$, where $p_{1}, p_{2}$ are the usual projection maps. The following question has been asked. If $X$ and $Y$ are two simple closed curves in the plane and $Y$ is contained in the bounded component of the plane minus $X$, then is the span of $X$ larger than the span of $Y$ ? We define a set of simple closed curves, which we refer to as being five star-like. We answer this question in the affirmative when $X$ is one of these simple closed curves. We calculate the spans of the simple closed curves in this collection and consider the spans of various geometric objects related to these simple closed curves.
\end{abstract}

\section{INTRODUCTION}

The span of a metric continuum was defined in 1964 by A. Lelek (see $[3$, p.209]). Variations of the span have been defined since then (cf $[4,5,2])$. In general it is difficult to evaluate the spans of a geometric object. It is of interest how these various spans, for a particular object, are related to each other. Also of interest is how the spans of related objects compare to each other. The following question by $\mathrm{H}$. Cook has been particularly interesting.

If $X_{1}$ and $X_{2}$ are two simple closed curves in the plane and $X_{2}$ is contained in the bounded component of $X_{1}$, then is the span of $X_{1}$ larger than the span of $X_{2}$ ? ([1])

This question has not yet been answered in general. Given some specific conditions on either $X_{1}$ or $X_{2}$, the answer has been shown to be yes. We

2000 Mathematics Subject Classification. 54F20, 54F15.

Key words and phrases. Span, simple closed curve. 
give a short summary of the results for these special cases that have been determined.

In the following results we assume that:

1. $X_{2}$ is a simple closed curve,

2. $X_{3}$ is a continuum that is contained in the bounded component of $R^{2}-X_{2}$,

3. $X_{1}$ is a plane separating continuum that contains $X_{2}$ in one of its bounded components, and

4. the span of $X$ is denoted by $\sigma(X)$.

If $X_{2}$ is the boundary of a convex region in the plane and $X_{1}$ is a simple closed curve, then $\sigma\left(X_{2}\right)<\sigma\left(X_{1}\right)$ ([7], see also [6]).

If $X_{2}$ is the boundary of a convex region in the plane, then $\sigma\left(X_{3}\right)<\sigma\left(X_{2}\right)<\sigma\left(X_{1}\right)[11]$.

Also, $\sigma\left(X_{3}\right)<\sigma\left(X_{2}\right)<\sigma\left(X_{1}\right)$ when $X_{2}$ is either an "indented circle" $([8,9,13])$, or a "concave upward symmetric" simple closed curve [10], or a simple closed curve as defined in [12]. In this paper we define a set of simple closed curves that we refer to as five star-like and show that if $X_{2}$ is a five star-like simple closed curve and $X_{1}$ and $X_{3}$ are as defined previously, then $\alpha\left(X_{3}\right)<\alpha\left(X_{2}\right)<\alpha\left(X_{1}\right)$, where $\alpha$ represents the span $\sigma$, semispan $\sigma_{0}$, surjective span $\sigma^{*}$, surjective semispan $\sigma_{0}^{*}$, symmetric span s, and surjective symmetric $\operatorname{span} s^{*}$.

\section{Preliminaries}

Let $X$ be a continuum, that is a compact, connected metric space. The span of $X, \sigma(X)$, is the least upper bound of the set of real numbers $r$ which satisfy the following conditions: there exist a continuum, $C$, and continuous functions $f, g: C \rightarrow X$, such that

$$
\operatorname{dmin}(f, g)=\min \{d(f(c), g(c)) \mid c \in C\} \geq r
$$

and

$$
\begin{gathered}
f(C)=g(C) \quad \sigma \\
\operatorname{span}
\end{gathered}
$$

To obtain the various other spans, we replace the preceeding equation with the following:

$$
\begin{gathered}
f(C) \subseteq g(C) \\
\text { semispan } \\
f(C)=g(C)=X \\
\text { surjective span } \\
f(C) \subseteq g(C)=X \\
\text { surjective semispan } \\
f(C)=g(C)
\end{gathered}
$$


symmetric span

and $\forall c \in C, \exists c^{\prime} \quad$ such that $f(c)=g\left(c^{\prime}\right)$ and $f\left(c^{\prime}\right)=g(c)$

$$
f(C)=g(C)=X \quad s^{*}
$$

surjective symmetric span

and $\forall c \in C, \exists c^{\prime} \quad$ such that $f(c)=g\left(c^{\prime}\right)$ and $f\left(c^{\prime}\right)=g(c)$.

The inequalities below follow immediately from the definitions,

$$
\begin{gathered}
0 \leq \sigma^{*}(X) \leq \sigma(X) \leq \sigma_{0}(X) \leq \operatorname{diam} X \\
0 \leq \sigma^{*}(X) \leq \sigma_{0}^{*}(X) \leq \sigma_{0}(X) \leq \operatorname{diam} X, \\
0 \leq s(X) \leq \sigma(X) \\
0 \leq s^{*}(X) \leq \sigma^{*}(X)
\end{gathered}
$$

The following results are easy consequences of the various definitions.

1. If $\mathrm{J}$ is an arc, then $\alpha(J)=0$, where $\alpha=\sigma, \sigma_{0}, \sigma^{*}, \sigma_{0}^{*}, s, s^{*}$.

2. If $\mathrm{X}$ is a simple closed curve, then $\sigma(X)=\sigma^{*}(X), \sigma_{0}(X)=\sigma_{0}^{*}(X)$, and $s(X)=s^{*}(X)$.

We utilize the following theorem from [3] in the proof of Corollary 2.

Theorem L: If $Y$ is a closed subset of the Hilbert cube $I^{\omega}$ and $\rho: Y \rightarrow S$ is an essential mapping of $Y$ onto the circumference $S$, then

$$
\inf _{s \in S}\left(\rho^{-1}(s), \rho^{-1}(-s)\right) \leq \sigma(Y) .
$$

\section{Main Results}

(a) Let $Q$ be a five-sided, convex polygon with sequentially labeled vertices $Q_{i}^{\prime}$ for $i=0,1,2,3,4$, with all interior angles $\angle Q_{i}^{\prime}, i=0,1,2,3,4$, larger than $90^{\circ}$. Extend each side $\overline{Q_{j}^{\prime} Q_{j+1}^{\prime}}$ of the polygon.

(b) Let $Q_{j+2}$ be the point of intersection of $\overleftrightarrow{Q_{j}^{\prime} Q_{j+1}^{\prime}}$ and $\overleftrightarrow{Q_{j+2}^{\prime} Q_{j+3}^{\prime}}$ for $j=0,1,2,3,4$, where all indices are taken modulo 5 .

(c) Let $X$ be the star shaped simple closed curve defined by $X=$ $\cup_{j=0}^{4}\left(\overline{Q_{j}^{\prime} Q_{j+1}} \cup \overline{Q_{j+1}^{\prime} Q_{j+1}}\right)$. We refer to $X$ as a five star-like simple closed curve.

(d) Let $t_{j}$ be the point on $\overline{Q_{j-1} Q_{j-3}}$ that is the closest to $Q_{j}$. Note that $t_{j} \in\left(\overline{Q_{j-2}^{\prime} Q_{j-1}}-\left\{Q_{j-2}^{\prime}\right\}\right)$, since $Q_{j} \in \overline{Q_{j-2}^{\prime} Q_{j-1}^{\prime}}$ and $\angle Q_{j-2}^{\prime}>90^{\circ}$.

(e) Let $r_{j}$ be the point on $\overline{Q_{j+1} Q_{j+3}}$ that is closest to $Q_{j}$. Note that $r_{j} \in\left(\overrightarrow{Q_{j+1} Q_{j+1}^{\prime}}-\left\{Q_{j+1}^{\prime}\right\}\right)$, since $Q_{j} \in \overrightarrow{Q_{j+1}^{\prime} Q_{j}^{\prime}}$ and $\angle Q_{j+1}^{\prime}>90^{\circ}$.

(f) Note that $t_{j}^{\prime}=d\left(Q_{j}, t_{j}\right)<d\left(Q_{j}, Q_{j+2}^{\prime}\right)=q_{j}^{\prime}$ and $r_{j}^{\prime}=d\left(Q_{j}, r_{j}\right)<$ $d\left(Q_{j}, Q_{j+2}^{\prime}\right)=q_{j}^{\prime}$. 
(g) Suppose that $q_{3}^{\prime}=\min \left\{q_{i}^{\prime}\right\}_{i=0}^{4}$. We can make this assumption since we can relabel the vertices so that this is true. We refer to the number

$$
\begin{array}{r}
\operatorname{fss}(X)=\max \left\{q_{3}^{\prime}, \min \left\{r_{0}^{\prime}, r_{1}^{\prime}, t_{1}^{\prime}, t_{2}^{\prime}\right\}, \min \left\{r_{1}^{\prime}, t_{2}^{\prime}, q_{4}^{\prime}\right\},\right. \\
\left.\min \left\{r_{4}^{\prime}, r_{0}^{\prime}, t_{0}^{\prime}, t_{1}^{\prime}\right\}, \min \left\{r_{4}^{\prime}, t_{0}^{\prime}, q_{2}^{\prime}\right\}\right\}
\end{array}
$$

as the five star-like spread of $X$, where $X$ is a five star-like simple closed curve.

TheOREM 3.1. Let $X$ be a five star-like simple closed curve. Then $\sigma(X)=$ $\sigma_{0}(X)=\sigma^{*}(X)=\sigma_{0}^{*}(X)=s(X)=s^{*}(X)=\mathrm{fss}(X)$.

Proof. Let $f, g: I \rightarrow X$ be continuous functions from $I$, the unit interval, onto $X$, such that their movements are always clockwise on $X$ and one function is constantly $Q_{j}$ for some $j$, while the other function moves from $Q_{k}$ to $Q_{k+1}$, passing through the point $Q_{k}^{\prime}$, where $k \neq j, j-1$ and $f[I]=g[I]=X$. Let $\mathcal{P}$ be the set consisting of all pairs of functions $(f, g)$ that satisfy these conditions. We claim that $\alpha(X)=\max \{\operatorname{dmin}(f, g) \mid(f, g) \in \mathcal{P}\}=\operatorname{fss}(X)$, where $\alpha=\sigma, \sigma_{0}, \sigma^{*}, \sigma_{0}^{*}, s, s^{*}$. We consider two cases.

CAse A: $\max \{\operatorname{dmin}(f, g) \mid(f, g) \in \mathcal{P}\}=q_{3}^{\prime}$

Consider the pair of functions given in Table 1.

TABLE 1. $f(t)$ and $g(t)$ versus t.

\begin{tabular}{|c|c|c|}
\hline$t$ & $f(t)$ & $g(t)$ \\
\hline 0 & $Q_{0}$ & $Q_{3}$ \\
\hline 0.1 & $Q_{1}$ & $Q_{3}$ \\
\hline 0.2 & $Q_{1}$ & $Q_{4}$ \\
\hline 0.3 & $Q_{2}$ & $Q_{4}$ \\
\hline 0.4 & $Q_{2}$ & $Q_{0}$ \\
\hline 0.5 & $Q_{3}$ & $Q_{0}$ \\
\hline 0.6 & $Q_{3}$ & $Q_{1}$ \\
\hline 0.7 & $Q_{4}$ & $Q_{1}$ \\
\hline 0.8 & $Q_{4}$ & $Q_{2}$ \\
\hline 0.9 & $Q_{0}$ & $Q_{2}$ \\
\hline 1.0 & $Q_{0}$ & $Q_{3}$ \\
\hline
\end{tabular}

Note that

$$
d\left(Q_{j}, \overline{Q_{j+2} Q_{j+2}^{\prime}} \cup \overline{Q_{j+2}^{\prime} Q_{j+3}}\right)=d\left(Q_{j}, Q_{j+2}^{\prime}\right)=q_{j}^{\prime} .
$$

Given this observation and assumption (g) in the construction of $X$, we see that $\operatorname{dmin}(f, g)=q_{3}^{\prime}$.

We observe that $\alpha(X) \geq d\left(Q_{3}, Q_{0}^{\prime}\right)=q_{3}^{\prime}$ for $\alpha=\sigma, \sigma_{0}, \sigma^{*}, \sigma_{0}^{*}, s, s^{*}$. This is true since, $\sigma(X)=\sigma^{*}(X), \sigma_{0}(X)=\sigma_{0}^{*}(X), f(I)=g(I)=X$, and for all $t \in[0,1]$ there is a $t^{\prime} \in[0,1]$ such that $f(t)=g\left(t^{\prime}\right)$ and $g(t)=f\left(t^{\prime}\right)$. 
In particular, $(f, g)$, from Table 1 , is a "better" pair than the pairs $\left(f_{1}, g_{1}\right)$ and $\left(f_{2}, g_{2}\right)$ given in Table 2 and Table 3.

TABLE 2. $f_{1}(t)$ and $g_{1}(t)$ versus $t$.

\begin{tabular}{|c|c|c|}
\hline$t$ & $f_{1}(t)$ & $g_{1}(t)$ \\
\hline 0 & $Q_{0}$ & $Q_{3}$ \\
\hline 0.1 & $Q_{0}$ & $Q_{4}$ \\
\hline 0.2 & $Q_{1}$ & $Q_{4}$ \\
\hline 0.3 & $Q_{2}$ & $Q_{4}$ \\
\hline 0.4 & $Q_{2}$ & $Q_{0}$ \\
\hline 0.5 & $Q_{3}$ & $Q_{0}$ \\
\hline 0.6 & $Q_{4}$ & $Q_{0}$ \\
\hline 0.7 & $Q_{4}$ & $Q_{1}$ \\
\hline 0.8 & $Q_{4}$ & $Q_{2}$ \\
\hline 0.9 & $Q_{0}$ & $Q_{2}$ \\
\hline 1.0 & $Q_{0}$ & $Q_{3}$ \\
\hline
\end{tabular}

TABLE 3. $f_{2}(t)$ and $g_{2}(t)$ versus $t$.

\begin{tabular}{|c|c|c|}
\hline$t$ & $f_{2}(t)$ & $g_{2}(t)$ \\
\hline 0 & $Q_{0}$ & $Q_{2}$ \\
\hline 0.1 & $Q_{1}$ & $Q_{2}$ \\
\hline 0.2 & $Q_{1}$ & $Q_{3}$ \\
\hline 0.3 & $Q_{1}$ & $Q_{4}$ \\
\hline 0.4 & $Q_{2}$ & $Q_{4}$ \\
\hline 0.5 & $Q_{2}$ & $Q_{0}$ \\
\hline 0.6 & $Q_{2}$ & $Q_{1}$ \\
\hline 0.7 & $Q_{3}$ & $Q_{1}$ \\
\hline 0.8 & $Q_{4}$ & $Q_{1}$ \\
\hline 0.9 & $Q_{4}$ & $Q_{2}$ \\
\hline 1.0 & $Q_{0}$ & $Q_{2}$ \\
\hline
\end{tabular}

Consequently, since

$$
\operatorname{dmin}(f, g) \geq \max \left\{\operatorname{dmin}\left(f_{1}, g_{1}\right), \operatorname{dmin}\left(f_{2}, g_{2}\right)\right\}
$$

it must be the case that

$$
\operatorname{dmin}(f, g)=q_{3}^{\prime} \geq \min \left\{r_{4}^{\prime}, t_{0}^{\prime}\right\}
$$

and

$$
\operatorname{dmin}(f, g)=q_{3}^{\prime} \geq \min \left\{t_{2}^{\prime}, r_{1}^{\prime}\right\}
$$


This implies that fss $(X)=q_{3}^{\prime}$.

We define a continuous function $p_{j}: Y \rightarrow \overrightarrow{Q_{j}^{\prime} Q_{j}} \cup \overrightarrow{Q_{j}^{\prime} Q_{j+1}}$ where $j=$ $0,1,2,3,4$, and $Y$ is the closure of the bounded component of $R^{2}-X$. First we define $p_{j}$ on $Y \cap Y_{l}$ where $Y_{l}$ is the closure of the bounded component of $R^{2}-\left(\overline{Q_{j}^{\prime} Q_{j+1}} \cup \overline{Q_{j+1} Q_{j+1}^{\prime}} \cup \overline{Q_{j+1}^{\prime} Q_{j+2}} \cup \overline{Q_{j+2} Q_{j+2}^{\prime}} \cup \overline{Q_{j+2}^{\prime} Q_{j+3}} \cup \overline{Q_{j+3} Q_{j}^{\prime}}\right)$. We consider two cases in defining $p_{j}$ on $Y_{l}$ for $j=0,1,2,3,4$.

CASE $p_{j}, Y_{l}, 1: t_{j+2}^{\prime} \leq r_{j+1}^{\prime}$

Let $t \in \overline{t_{j+2} Q_{j+1}}$ and let $L_{t}$ be the line that is parallel to $\overline{Q_{j+2} t_{j+2}}$ and passes through the point t. For each $y \in Y \cap L_{t}$, let $p_{j}(y)=t$. It may be the case $Q_{j+1}=t_{j+2}$. If so, then $L_{t} \cap Y=\left\{Q_{j+1}, Q_{j+2}\right\}$ and $\operatorname{diam}\left(p_{j}^{-1}\{t\}\right)=$ $d\left(Q_{j+2}^{\prime}, t_{j+2}\right)=t_{j+2}^{\prime}$. Otherwise, $\overline{Q_{j+1} t_{j+2}} \cup \overline{t_{j+2} Q_{j+2}} \cup \overline{Q_{j+2} Q_{j+1}}$ forms a right triangle and for each $\mathrm{t}, \operatorname{diam}\left(p_{j}^{-1}\{t\}\right) \leq t_{j+2}^{\prime}$.

Let $h_{1}:[0,1] \rightarrow \overline{Q_{j}^{\prime} t_{j+2}}$ be the homeomorphism such that $h_{1}(t)=(1-$ $t) t_{j+2}+t Q_{j}^{\prime}$.

Let $h_{2}:[0,1] \rightarrow \overline{Q_{j+2} Q_{j+3}}$ be the homeomorphism such that $h_{2}(t)=$ $(1-t) Q_{j+2}+t Q_{j+3}$.

Let $L_{t}$ be the line connecting $h_{1}(t)$ and $h_{2}(t)$ for each $t \in[0,1]$. For each $y \in Y \cap L_{t}$ let $p_{j}(y)=h_{1}(t)$. Note that $\operatorname{diam}\left(p_{j}^{-1}\left(\left\{h_{1}(t)\right\}\right)\right) \leq$ $\max \left\{t_{j+2}^{\prime}, q_{j+3}^{\prime}\right\}$.

CASE $p_{j}, Y_{l}, 2: r_{j+1}^{\prime}<t_{j+2}^{\prime}$

Let $t \in \overline{r_{j+1} Q_{j+2}}$ and let $L_{t}$ be the line that is parallel to $\overline{r_{j+1} Q_{j+1}}$ and passes through the point t. Let $t^{\prime}$ be the point such that $\left\{t^{\prime}\right\}=L_{t} \cap$ $\left(\left(\overrightarrow{Q_{j}^{\prime} Q_{j+1}}-\overline{Q_{j}^{\prime} Q_{j+1}}\right) \cup Q_{j+1}\right)$. For each $y \in Y \cap L_{t}$ let $p_{j}(y)=t^{\prime}$. Note that for each $t^{\prime}, \operatorname{diam}\left(p_{j}^{-1}\left\{t^{\prime}\right\}\right) \leq r_{j+1}^{\prime}$.

Let $h_{1}:[0,1] \rightarrow \overline{Q_{j}^{\prime} Q_{j+1}}$ be the homeomorphism such that $h_{1}(t)=(1-$ t) $Q_{j+1}+t Q_{j}^{\prime}$.

Let $h_{2}:[0,1] \rightarrow \overline{r_{j+1} Q_{j+3}}$ be the homeomorphism such that $h_{2}(t)=$ $(1-t) r_{j}+t Q_{j+3}$.

Let $L_{t}$ be the line connecting $h_{1}(t)$ and $h_{2}(t)$. For each $y \in L_{t} \cap Y$ let $p_{j}(y)=h_{1}(t)$. Note that for each $\mathrm{t}, \operatorname{diam}\left(p_{j}^{-1}\left(\left\{h_{1}(t)\right\}\right)\right) \leq \max \left\{r_{j+1}^{\prime}, q_{j+3}^{\prime}\right\}$.

The definition of $p_{j}$ on $Y \cap Y_{r}$, where $Y_{r}$ is the closure of the bounded component of $R^{2}-\left(\overline{Q_{j}^{\prime} Q_{j}} \cup \overline{Q_{j} Q_{j+4}^{\prime}} \cup \overline{Q_{j+4}^{\prime} Q_{j+4}} \cup \overline{Q_{j+4} Q_{j+3}^{\prime}} \cup \overline{Q_{j+3}^{\prime} Q_{j+3}} \cup\right.$ $\left.\overline{Q_{j+3} Q_{j}^{\prime}}\right)$ is defined in a similar manner in the two corresponding cases.

CASE $p_{j}, Y_{r}, 1: r_{j+4}^{\prime} \leq t_{j}^{\prime}$.

In this case for $y \in Y \cap Y_{r}, \operatorname{diam}\left(p_{j}^{-1}\left(\left\{p_{j}(y)\right\}\right)\right) \leq \max \left\{r_{j+4}^{\prime}, q_{j+3}^{\prime}\right\}$

CASE $p_{j}, Y_{r}, 2: t_{j}^{\prime}<r_{j+4}^{\prime}$

In this case for $y \in Y \cap Y_{r}$, $\operatorname{diam}\left(p_{j}^{-1}\left(\left\{p_{j}(y)\right\}\right)\right) \leq \max \left\{t_{j}^{\prime}, q_{j+3}^{\prime}\right\}$. 
We see that for $y \in Y_{l}, \operatorname{diam}\left(p_{j}^{-1}\left(\left\{p_{j}(y)\right\}\right)\right) \leq \max \left\{\min \left\{r_{j+1}^{\prime}, t_{j+2}^{\prime}\right\}, q_{j+3}^{\prime}\right\}$. For $y \in Y_{r}, \operatorname{diam}\left(p_{j}^{-1}\left(\left\{p_{j}(y)\right\}\right)\right) \leq \max \left\{\min \left\{r_{j+4}^{\prime}, t_{j}^{\prime}\right\}, q_{j+3}^{\prime}\right\}$. Consequently, for $y \in Y$,

$$
\operatorname{diam}\left(p_{j}^{-1}\left(\left\{p_{j}(y)\right\}\right)\right) \leq \max \left\{\min \left\{r_{j+1}^{\prime}, t_{j+2}^{\prime}\right\}, \min \left\{r_{j+4}^{\prime}, t_{j}^{\prime}\right\}, q_{j+3}^{\prime}\right\} .
$$

Let $f^{*}, g^{*}: C \rightarrow Z$ be any two continuous functions from a continuum $C$ into a continuum $Z \subseteq Y$, such that $f^{*}(C) \subseteq g^{*}(C) \subseteq Z$. Consider $p_{j} \circ$ $f^{*}, p_{j} \circ g^{*}: C \rightarrow\left(\overrightarrow{Q_{j}^{\prime} Q_{j}} \cup \overrightarrow{Q_{j}^{\prime} Q_{j+1}}\right)$. The image of $p_{j} \circ g^{*}(C)$ is an arc and $p_{j} \circ f^{*}(C) \subseteq p_{j} \circ g^{*}(C)$. Since all the spans of an arc are zero, there is a $c \in C$ such that $p_{j} \circ f^{*}(c)=p_{j} \circ g^{*}(c)$. Consequently, $d\left(f^{*}(c), g^{*}(c)\right) \leq$ $\operatorname{diam}\left(p_{j}^{-1}\left\{p_{j}\left(g^{*}(c)\right)\right\}\right) \leq \max \left\{\min \left\{r_{j+1}^{\prime}, t_{j+2}^{\prime}\right\}, \min \left\{r_{j+4}^{\prime}, t_{j}^{\prime}\right\}, q_{j+3}^{\prime}\right\}$ and

$$
\sigma_{0}(Z) \leq \max \left\{\min \left\{r_{j+1}^{\prime}, t_{j+2}^{\prime}\right\}, \min \left\{r_{j+4}^{\prime}, t_{j}^{\prime}\right\}, q_{j+3}^{\prime}\right\} .
$$

In this case (i.e. case A) when $\operatorname{fss}(X)=\operatorname{dmin}(f, g)$ where $f$ and $g$ are defined in Table 1 , we conclude that $\sigma_{0}(X) \leq q_{j+3}^{\prime}$ by taking $Z=X \subseteq Y$, $j=0$, and using $(*)$. Given the inequalities relating the various spans and the fact that for each $t \in[0,1]$ there is a $t^{\prime} \in[0,1]$ such that $g(t)=f\left(t^{\prime}\right)$ and $f(t)=g\left(t^{\prime}\right)$, we conclude that $\sigma(X)=\sigma_{0}(X)=\sigma^{*}(X)=\sigma_{0}^{*}(X)=s(X)=$ $s^{*}(X)=\operatorname{fss}(X)=q_{3}^{\prime}$. This completes case A.

CASE B: $\max \{\operatorname{dmin}(f, g) \mid(f, g) \in \mathcal{P}\}>q_{3}^{\prime}$

Let $(f, g) \in \mathcal{P}$ such that $\max \{\operatorname{dmin}(f, g) \mid(f, g) \in \mathcal{P}\}=\operatorname{dmin}(f, g)>q_{3}^{\prime}$. Since $r_{3}^{\prime}<q_{3}^{\prime}$ and $t_{3}^{\prime}<q_{3}^{\prime}$, the pair $(f, g)$ can not include the following (forbidden) steps as given in Table 4 and Table 5.

TABLE 4. Forbidden steps

\begin{tabular}{|c|c|c|}
\hline$t$ & $f(t)$ & $g(t)$ \\
\hline$t_{1}$ & $Q_{4}$ & $Q_{3}$ \\
\hline$t_{2}$ & $Q_{0}$ & $Q_{3}$ \\
\hline$t_{1}$ & $Q_{0}$ & $Q_{3}$ \\
\hline$t_{2}$ & $Q_{1}$ & $Q_{3}$ \\
\hline
\end{tabular}

TABLE 5. Forbidden steps

\begin{tabular}{|c|c|c|}
\hline$t$ & $f(t)$ & $g(t)$ \\
\hline$t_{1}$ & $Q_{3}$ & $Q_{4}$ \\
\hline$t_{2}$ & $Q_{3}$ & $Q_{0}$ \\
\hline
\end{tabular} \begin{tabular}{|c|c|c|}
\hline$t$ & $f(t)$ & $g(t)$ \\
\hline$t_{1}$ & $Q_{3}$ & $Q_{0}$ \\
\hline$t_{2}$ & $Q_{1}$ \\
\hline$t_{1}$ & $Q_{3}$ & $Q_{1}$ \\
\hline$t_{2}$ & $Q_{3}$ & $Q_{2}$ \\
\hline
\end{tabular}

For any pair of functions, $f$ and $g$, we consider all the possible starting values of $f$ and $g$, that is $f(0)$ and $g(0)$, and determine what steps are possible for $t>0$ given the restriction $\operatorname{dmin}(f, g)>d\left(Q_{3}, Q_{1}^{\prime}\right)=q_{3}^{\prime}$. Because of the symmetry of this process, we consider either $f(0)=Q_{j}$ and $g(0)=Q_{k}$ where $k \neq j$, or $f(0)=Q_{k}$ and $g(0)=Q_{j}$ for $k \neq j$, but not both. All 
these possibilities are summarized in the tables below. For $n \in N$, we let $0=t_{0}<t_{1}<t_{2}<\cdots<t_{n}<1$.

TABLE 6. Left to right: Patterns I, II, III, IV .

\begin{tabular}{|c|c|c|}
\hline$t$ & $f(t)$ & $g(t)$ \\
\hline 0 & $Q_{0}$ & $Q_{2}$ \\
\hline$t_{1}$ & $Q_{1}$ & $Q_{2}$ \\
\hline$t_{2}$ & $Q_{1}$ & $Q_{3}$ \\
\hline$t_{3}$ & $Q_{1}$ & $Q_{4}$ \\
\hline$t_{4}$ & $Q_{1}$ & $Q_{0}$ \\
\hline$t_{5}$ & $Q_{2}$ & $Q_{0}$ \\
\hline
\end{tabular}

\begin{tabular}{|c|c|c|}
\hline$t$ & $f(t)$ & $g(t)$ \\
\hline 0 & $Q_{0}$ & $Q_{2}$ \\
\hline$t_{1}$ & $Q_{1}$ & $Q_{2}$ \\
\hline$t_{2}$ & $Q_{1}$ & $Q_{3}$ \\
\hline$t_{3}$ & $Q_{1}$ & $Q_{4}$ \\
\hline$t_{4}$ & $Q_{2}$ & $Q_{4}$ \\
\hline$t_{5}$ & $Q_{2}$ & $Q_{0}$ \\
\hline
\end{tabular}

\begin{tabular}{|c|c|c|}
\hline$t$ & $f(t)$ & $g(t)$ \\
\hline 0 & $Q_{0}$ & $Q_{2}$ \\
\hline$t_{1}$ & $Q_{0}$ & $Q_{3}$ \\
\hline$t_{2}$ & $Q_{0}$ & $Q_{4}$ \\
\hline$t_{3}$ & $Q_{1}$ & $Q_{4}$ \\
\hline$t_{4}$ & $Q_{1}$ & $Q_{0}$ \\
\hline$t_{5}$ & $Q_{2}$ & $Q_{0}$ \\
\hline
\end{tabular}

\begin{tabular}{|c|c|c|}
\hline$t$ & $f(t)$ & $g(t)$ \\
\hline 0 & $Q_{0}$ & $Q_{2}$ \\
\hline$t_{1}$ & $Q_{0}$ & $Q_{3}$ \\
\hline$t_{2}$ & $Q_{0}$ & $Q_{4}$ \\
\hline$t_{3}$ & $Q_{1}$ & $Q_{4}$ \\
\hline$t_{4}$ & $Q_{2}$ & $Q_{4}$ \\
\hline$t_{5}$ & $Q_{2}$ & $Q_{0}$ \\
\hline
\end{tabular}

TABLE 7 .

\begin{tabular}{|c|c|c|}
\hline$t$ & $f(t)$ & $g(t)$ \\
\hline 0 & $Q_{0}$ & $Q_{1}$ \\
\hline$t_{1}$ & $Q_{0}$ & $Q_{2}$ \\
\hline
\end{tabular}

TABLE 8. Left to right: Patterns V and VI.

\begin{tabular}{|c|c|c|}
\hline$t$ & $f(t)$ & $g(t)$ \\
\hline 0 & $Q_{4}$ & $Q_{0}$ \\
\hline$t_{1}$ & $Q_{4}$ & $Q_{1}$ \\
\hline$t_{2}$ & $Q_{4}$ & $Q_{2}$ \\
\hline$t_{3}$ & $Q_{0}$ & $Q_{2}$ \\
\hline$t_{1}$ & $Q_{4}$ & $Q_{4}$ \\
\hline$t_{2}$ & $Q_{0}$ & $Q_{1}$ \\
\hline$t_{3}$ & $Q_{0}$ & $Q_{2}$ \\
\hline
\end{tabular}

TABLE 9.

\begin{tabular}{|c|c|c|}
\hline$t$ & $f(t)$ & $g(t)$ \\
\hline 0 & $Q_{3}$ & $Q_{0}$ \\
\hline$t_{1}$ & $Q_{4}$ & $Q_{0}$ \\
\hline
\end{tabular}

We start with $f(0)=Q_{0}$ and $g(0)=Q_{2}$ in Table 6 . We see that there are four possibilities: pattern I, II, III, or IV. In Table 7 , where $f(0)=Q_{0}$ and $g(0)=Q_{1}$, there is only one possible second step. After this step, the functions would follow one of the patterns I, II, III, or IV in Table 6. In Table 8, we start with $f(0)=Q_{4}$ and $g(0)=Q_{0}$. We see that there are two possibilities, patterns V and VI. Each of these ends with $f\left(t_{3}\right)=Q_{0}$ and $g\left(t_{3}\right)=Q_{2}$. These functions would then follow either pattern I, II, III, or IV 
TABLE 10. Left to right: Patterns VII and VIII.

\begin{tabular}{|c|c|c|}
\hline$t$ & $f(t)$ & $g(t)$ \\
\hline 0 & $Q_{4}$ & $Q_{1}$ \\
\hline$t_{1}$ & $Q_{4}$ & $Q_{2}$ \\
\hline$t_{2}$ & $Q_{0}$ & $Q_{2}$ \\
\hline 0 & $f(t)$ & $g(t)$ \\
\hline$t_{1}$ & $Q_{4}$ & $Q_{1}$ \\
\hline$t_{2}$ & $Q_{0}$ & $Q_{2}$ \\
\hline
\end{tabular}

TABle 11.

\begin{tabular}{|c|c|c|}
\hline$t$ & $f(t)$ & $g(t)$ \\
\hline 0 & $Q_{2}$ & $Q_{1}$ \\
\hline$t_{1}$ & $Q_{3}$ & $Q_{1}$ \\
\hline$t_{2}$ & $Q_{4}$ & $Q_{1}$ \\
\hline
\end{tabular}

TABLE 12.

\begin{tabular}{|c|c|c|}
\hline$t$ & $f(t)$ & $g(t)$ \\
\hline 0 & $Q_{3}$ & $Q_{1}$ \\
\hline$t_{1}$ & $Q_{4}$ & $Q_{1}$ \\
\hline
\end{tabular}

TABLE 13.

\begin{tabular}{|c|c|c|}
\hline$t$ & $f(t)$ & $g(t)$ \\
\hline 0 & $Q_{3}$ & $Q_{2}$ \\
\hline$t_{1}$ & $Q_{4}$ & $Q_{2}$ \\
\hline$t_{2}$ & $Q_{0}$ & $Q_{2}$ \\
\hline
\end{tabular}

in Table 6. In Table 9 we have $f(0)=Q_{3}$ and $g(0)=Q_{0}$. There is only one possible second step, that is $f\left(t_{1}\right)=Q_{4}$ and $g\left(t_{1}\right)=Q_{0}$. These functions then would follow either pattern V or VI in Table 8. After this, the functions would follow one of the patterns, I, II, III, or IV in Table 6. In Table 10, we have $f(0)=Q_{4}$ and $g(0)=Q_{1}$. There are two possibilities, patterns VII or VIII. Both of these patterns end with $f\left(t_{2}\right)=Q_{0}$ and $g\left(t_{2}\right)=Q_{2}$. These functions would then follow one of the patterns I, II, III, or IV in Table 6. Similarly, the functions in Table 11 would subsequently follow one of the patterns VII or VIII in Table 10, then follow one of the patterns I, II, III, or IV in Table 6. The functions in Table 12 would follow either pattern VII or VIII in Table 10, then either pattern I, II, III, or IV in Table 6. The pairs of functions in tables 13 and 14 would each follow one of the patterns I, II, III, or IV in Table 6.

We have considered all possible combinations for $f(0)$ and $g(0)$. Note that $f(0)=Q_{3}$ and $g(0)=Q_{4}$ is not possible, since for $t_{1}, f\left(t_{1}\right)=Q_{3}, g\left(t_{1}\right)=Q_{0}$, and $\operatorname{dmin}(f, g) \leq r_{3}<d\left(Q_{3}, Q_{0}^{\prime}\right)$. We see that all pairs of functions $f$ and $g$, satisfying our conditions, contain one of the patterns I, II, III, or IV. So, if 
TABLE 14.

\begin{tabular}{|c|c|c|}
\hline$t$ & $f(t)$ & $g(t)$ \\
\hline 0 & $Q_{4}$ & $Q_{2}$ \\
\hline$t_{1}$ & $Q_{0}$ & $Q_{2}$ \\
\hline
\end{tabular}

there is a pair of functions $(f, g) \in \mathcal{P}$ such that $\operatorname{dmin}(f, g)>d\left(Q_{3}, Q_{0}^{\prime}\right)=q_{3}^{\prime}$, then $f$ and $g$ contain one of the patterns I, II, III, or IV. So, for any pair, $(f, g) \in \mathcal{P}$, we are now considering, we see that:

$$
\operatorname{dmin}(f, g) \leq \max \{\min \text { I, min II, min III, min IV }\}
$$

where

$$
\begin{aligned}
d\left(Q_{i}, t_{i}\right) & =t_{i}^{\prime}, \quad d\left(Q_{i}, r_{i}\right)=r_{i}^{\prime}, \quad d\left(Q_{i}, Q_{i+2}^{\prime}\right)=q_{i}^{\prime}, \quad \text { and } \\
\min \mathrm{I} & =\min \left\{r_{0}^{\prime}, r_{1}^{\prime}, t_{1}^{\prime}, t_{2}^{\prime}, q_{1}^{\prime}\right\}=\min \left\{r_{0}^{\prime}, r_{1}^{\prime}, t_{1}^{\prime}, t_{2}^{\prime}\right\} \\
\min \mathrm{II} & =\min \left\{r_{1}^{\prime}, t_{2}^{\prime}, q_{1}^{\prime}, q_{2}^{\prime}, q_{4}^{\prime}\right\}=\min \left\{r_{1}^{\prime}, t_{2}^{\prime}, q_{4}^{\prime}\right\} \\
\min \mathrm{III} & =\min \left\{r_{0}^{\prime}, r_{4}^{\prime}, t_{0}^{\prime}, t_{1}^{\prime}, q_{0}^{\prime}\right\}=\min \left\{r_{0}^{\prime}, r_{4}^{\prime}, t_{0}^{\prime}, t_{1}^{\prime}\right\} \\
\min \mathrm{IV} & =\min \left\{r_{4}^{\prime}, t_{0}^{\prime}, q_{0}^{\prime}, q_{2}^{\prime}, q_{4}^{\prime}\right\}=\min \left\{r_{4}^{\prime}, t_{0}^{\prime}, q_{2}^{\prime}\right\} .
\end{aligned}
$$

Note that patterns I and III are mirror images of each other, as are II and IV.

If the "best" $f$ and $g$ is not as given in Table 1, then since all other pairs, $f$ and $g$, contain one of the patterns I, II, III, or IV, then the best $f$ and $g$ must be one of the pairs of functions given in Table 15. Hence, the "best" pair $f$ and $g$ will be from either Table I, II, III, or IV in Table 15, and

$$
\operatorname{dmin}(f, g)=\max \{\min \mathrm{I}, \min \mathrm{II}, \min \mathrm{III}, \min \mathrm{IV}\},
$$

where $\operatorname{dmin}(f, g)=\min$ I for Table I, $\operatorname{dmin}(f, g)=\min$ II for Table II, $\operatorname{dmin}(f, g)=\min$ III for Table III, and $\operatorname{dmin}(f, g)=\min$ IV for Table IV. Consequently, $\alpha(X) \geq \max \{\min$ I, min II, min III, min IV $\}$, where $\alpha=$ $\sigma, \sigma_{0}, \sigma^{*} \sigma_{0}^{*}, s, s^{*}$ since the functions $f$ and $g$ satisfy the requirements for all of the spans. We will show that $\sigma_{0}(X) \leq \max \{\min$ I, min II, min III, min IV $\}$ and consequently that $\alpha(X)=\max \{\min$ I, min II, min III, min IV $\}$ for $\alpha=\sigma, \sigma_{0}, \sigma^{*}, \sigma_{0}^{*}, s, s^{*}$.

Since I and III are mirror images of each other as are II and IV, we need to examine only two cases,

$$
\min I=\max \{\min I, \min I I, \min I I I, \min I V\}
$$

and

$$
\min I I=\max \{\min I, \min I I, \min I I I, \min I V\}
$$

CASE 1: $\min$ II $=\max \{\min I, \min I I, \min I I I, \min I V\}=\min \left\{r_{1}^{\prime}, t_{2}^{\prime}, q_{4}^{\prime}\right\}$.

Suppose that $\max \{\min$ I, $\min$ II, $\min$ III, $\min I V\}=\min$ II, that is, the best pair of functions $f$ and $g$ is from Table II. So, $\operatorname{dmin}(f, g)=\min$ 
Table 15. Left to right Table I, Table II, Table III, Table IV.

\begin{tabular}{|c|c|c|c|c|c|c|c|c|c|c|c|}
\hline $\bar{t}$ & $f(t)$ & $g(t)$ & $t$ & $f(t)$ & $g(t)$ & $t$ & $f(t)$ & $g(t)$ & $t$ & $f(t)$ & $g(t)$ \\
\hline 0 & $Q_{0}$ & $Q_{2}$ & 0 & $Q_{0}$ & $Q_{2}$ & 0 & $Q_{0}$ & $Q_{2}$ & 0 & $Q_{0}$ & $Q_{2}$ \\
\hline 0.1 & $Q_{1}$ & $Q_{2}$ & 0.1 & $Q_{1}$ & $Q_{2}$ & 0.1 & $Q_{0}$ & $Q_{3}$ & 0.1 & $Q_{0}$ & $Q_{3}$ \\
\hline 0.2 & $Q_{1}$ & $Q_{3}$ & 0.2 & $Q_{1}$ & $Q_{3}$ & 0.2 & $Q_{0}$ & $Q_{4}$ & 0.2 & $Q_{0}$ & $Q_{4}$ \\
\hline 0.3 & $Q_{1}$ & $Q_{4}$ & 0.3 & $Q_{1}$ & $Q_{4}$ & 0.3 & $Q_{1}$ & $Q_{4}$ & 0.3 & $Q_{1}$ & $Q_{4}$ \\
\hline 0.4 & $Q_{1}$ & $Q_{0}$ & 0.4 & $Q_{2}$ & $Q_{4}$ & 0.4 & $Q_{1}$ & $Q_{0}$ & 0.4 & $Q_{2}$ & $Q_{4}$ \\
\hline 0.5 & $Q_{2}$ & $Q_{0}$ & 0.5 & $Q_{2}$ & $Q_{0}$ & 0.5 & $Q_{2}$ & $Q_{0}$ & 0.5 & $Q_{2}$ & $Q_{0}$ \\
\hline 0.6 & $Q_{2}$ & $Q_{1}$ & 0.6 & $Q_{2}$ & $Q_{1}$ & 0.6 & $Q_{3}$ & $Q_{0}$ & 0.6 & $Q_{3}$ & $Q_{0}$ \\
\hline 0.7 & $Q_{3}$ & $Q_{1}$ & 0.7 & $Q_{3}$ & $Q_{1}$ & 0.7 & $Q_{4}$ & $Q_{0}$ & 0.7 & $Q_{4}$ & $Q_{0}$ \\
\hline 0.8 & $Q_{4}$ & $Q_{1}$ & 0.8 & $Q_{4}$ & $\overline{Q_{1}}$ & 0.8 & $Q_{4}$ & $Q_{1}$ & 0.8 & $Q_{4}$ & $Q_{1}$ \\
\hline 0.9 & $Q_{0}$ & $Q_{1}$ & 0.9 & $Q_{4}$ & $Q_{2}$ & 0.9 & $Q_{0}$ & $Q_{1}$ & 0.9 & $Q_{4}$ & $Q_{2}$ \\
\hline 1.0 & $Q_{0}$ & $Q_{2}$ & 1.0 & $Q_{0}$ & $\overline{Q_{2}}$ & 1.0 & $Q_{0}$ & $Q_{2}$ & 1.0 & $Q_{0}$ & $Q_{2}$ \\
\hline
\end{tabular}

$\mathrm{II}=\min \left\{r_{1}^{\prime}, t_{2}^{\prime}, q_{4}^{\prime}\right\}$ and $\alpha(X) \geq \min \left\{r_{1}^{\prime}, t_{2}^{\prime}, q_{4}^{\prime}\right\}$, where $\alpha=\sigma, \sigma_{0}, \sigma^{*}, \sigma_{0}^{*}, s$, and $s^{*}$. We will now show that $\alpha(X)=\min \left\{r_{1}^{\prime}, t_{2}^{\prime}, q_{4}^{\prime}\right\}=\operatorname{dmin}(f, g)$, where $\alpha=\sigma, \sigma_{0}, \sigma^{*}, \sigma_{0}^{*}, s$, and $s^{*}$.

Let $f^{*}, g^{*}: C \rightarrow Z$ be continuous functions from a continuum $C$ into a continuum $Z \subseteq Y$ such that $f^{*}(C) \subseteq g^{*}(C)$. We consider three subcases.

CASE $1 \mathrm{~A}$ : $\min \mathrm{II}=r_{1}^{\prime}, r_{1}^{\prime} \leq t_{2}^{\prime}$, and $r_{1}^{\prime} \leq q_{4}^{\prime}$.

Observe that $\min \mathrm{IV}=\min \left\{r_{4}^{\prime}, t_{0}^{\prime}, q_{2}^{\prime}\right\} \leq \min \mathrm{II}=r_{1}^{\prime} \leq t_{2}^{\prime}<q_{2}^{\prime}$, so $\min \mathrm{IV} \neq q_{2}^{\prime}$ and $\min \mathrm{IV}=\min \left\{r_{4}^{\prime}, t_{0}^{\prime}\right\}$. So, either $r_{4}^{\prime} \leq r_{1}^{\prime}=\operatorname{dmin}(f, g)$ or $t_{0}^{\prime} \leq r_{1}^{\prime}=\operatorname{dmin}(f, g)$. We define $p_{0}: Y \rightarrow \overrightarrow{Q_{0}^{\prime}, Q_{0}} \cup \overrightarrow{Q_{0}^{\prime} Q_{1}}$ based on $r_{1}^{\prime} \leq t_{2}^{\prime}$ and either $r_{4}^{\prime} \leq r_{1}^{\prime}$ or $t_{0}^{\prime} \leq r_{1}^{\prime}$. Since $r_{1}^{\prime} \leq t_{2}^{\prime}$, we define $p_{0}$ on $Y_{l}$ by using case $p_{0}, Y_{l}$, 1 when $r_{1}^{\prime}=t_{2}^{\prime}$ and case $p_{0}, Y_{l}, 2$ when $r_{1}^{\prime}<t_{2}^{\prime}$. Also, either $r_{4}^{\prime} \leq t_{0}^{\prime}$ or $t_{0}^{\prime}<r_{4}^{\prime}$. We define $p_{0}$ on $Y_{r}$ by case $p_{0}, Y_{r}, 1$ when $r_{4}^{\prime} \leq t_{0}^{\prime}$ and by case $p_{0}, Y_{r}, 2$ when $t_{0}^{\prime}<r_{4}^{\prime}$. Consider $p_{0} \circ f^{*}, p_{0} \circ g^{*}: C \rightarrow \overrightarrow{Q_{0}^{\prime} Q_{0}} \cup \overrightarrow{Q_{0}^{\prime} Q_{1}}$. Since the range of $p \circ g^{*}$ is an interval and $p_{0} \circ f^{*}(C) \subset p_{0} \circ g^{*}(C)$, we see that there is a $c \in C$ such that $p_{0} \circ f^{*}(c)=p_{0} \circ g^{*}(c)$ and $d\left(f^{*}(c), g^{*}(c)\right) \leq \max \left\{\min \left\{t_{0}^{\prime}, r_{4}^{\prime}\right\}, q_{3}^{\prime}, r_{1}^{\prime}\right\}=r_{1}^{\prime}$.

CASE 1B: $\min \mathrm{II}=t_{2}^{\prime}, t_{2}^{\prime}<r_{1}^{\prime}$, and $t_{2}^{\prime} \leq q_{4}^{\prime}$.

Observe that $\min \mathrm{IV}=\min \left\{r_{4}^{\prime}, t_{0}^{\prime}, q_{2}^{\prime}\right\} \leq \min \mathrm{II}=t_{2}^{\prime}<q_{2}^{\prime}$, so $\min \mathrm{IV}$ $\neq q_{2}^{\prime}$, and $\min \mathrm{IV}=\min \left\{r_{4}^{\prime}, t_{0}^{\prime}\right\}$. So, either $r_{4}^{\prime} \leq t_{2}^{\prime}=\operatorname{dmin}(f, g)$ or $t_{0}^{\prime} \leq t_{2}^{\prime}=$ $\operatorname{dmin}(f, g)$. We define $p_{0}: Y \rightarrow \overrightarrow{Q_{0}^{\prime} Q_{0}} \cup \overrightarrow{Q_{0}^{\prime} Q_{1}}$ based on $t_{2}^{\prime}<r_{1}^{\prime}$ and either $r_{4}^{\prime} \leq t_{2}^{\prime}$ or $t_{0}^{\prime} \leq t_{2}^{\prime}$. Since $t_{2}^{\prime}<r_{1}^{\prime}$, we define $p_{0}$ on $Y_{l}$ by using case $p_{0}, Y_{l}, 1$. Also, either $r_{4}^{\prime} \leq t_{0}^{\prime}$ or $t_{0}^{\prime}<r_{4}^{\prime}$. We define $p_{0}$ on $Y_{r}$ by case $p_{0}, Y_{r}, 1$ when $r_{4}^{\prime} \leq t_{0}^{\prime}$ and by case $p_{0}, Y_{r}, 2$ when $t_{0}^{\prime}<r_{4}^{\prime}$. Again we conclude that there is a $c \in C$ such that $d\left(f^{*}(c), g^{*}(c)\right) \leq \max \left\{\min \left\{t_{0}^{\prime}, r_{4}^{\prime}\right\}, q_{3}^{\prime}, t_{2}^{\prime}\right\}=t_{2}^{\prime}$.

CASE $1 \mathrm{C}$ : $\min \mathrm{II}=q_{4}^{\prime}, q_{4}^{\prime}<r_{1}^{\prime}$, and $q_{4}^{\prime}<t_{2}^{\prime}$. 
Observe that $\min \mathrm{I}=\min \left\{r_{0}^{\prime}, r_{1}^{\prime}, t_{1}^{\prime}, t_{2}^{\prime}\right\} \leq \min \mathrm{II}=q_{4}^{\prime}<r_{1}^{\prime}$ and $q_{4}^{\prime}<t_{2}^{\prime}$, min $\mathrm{I}=\min \left\{r_{0}^{\prime}, t_{1}^{\prime}\right\}$, so either $r_{0}^{\prime} \leq q_{4}^{\prime}=\operatorname{dmin}(f, g)$ or $t_{1}^{\prime} \leq$ $q_{4}^{\prime}=\operatorname{dmin}(f, g)$. We define $p_{1}: Y \rightarrow \overrightarrow{Q_{1}^{\prime} Q_{1}} \cup \overrightarrow{Q_{1}^{\prime} Q_{2}}$ based on either $r_{0}^{\prime} \leq t_{1}^{\prime}$ or $t_{1}^{\prime}<r_{0}^{\prime}$ for $y \in Y_{r}$ and either $t_{3}^{\prime} \leq r_{2}^{\prime}$ or $r_{2}^{\prime}<t_{3}^{\prime}$ for $y \in Y_{l}$. Note that $t_{3}^{\prime}<q_{3}^{\prime}<q_{4}^{\prime}$. So we see that there is a $c \in C$ such that $d\left(f^{*}(c), g^{*}(c)\right) \leq \max \left\{\min \left\{r_{0}^{\prime}, t_{1}^{\prime}\right\},\left\{\min \left\{r_{2}^{\prime}, t_{3}^{\prime}\right\}, q_{4}^{\prime}\right\}=q_{4}^{\prime}\right.$.

Therefore, in case 1 we conclude that $\sigma_{0}(X) \leq \operatorname{dmin}(f, g)=\min$ II. Since $X$ is a simple closed curve we know that $\sigma(X)=\sigma^{*}(X)$ and $\sigma_{0}(X)=\sigma_{0}^{*}(X)$. Also, we see that for all $t \in[0,1]$ there is a $t^{\prime} \in[0,1]$ such that $f(t)=g\left(t^{\prime}\right)$ and $f\left(t^{\prime}\right)=g(t)$. Consequently, we see that $\sigma(X)=\sigma_{0}(X)=\sigma^{*}(X)=\sigma_{0}^{*}(X)=$ $s(X)=s^{*}(X)=\min$ II.

CASE 2: $\min I=\max \{\min I, \min I I, \min I I I, \min I V\}=\min \left\{r_{0}^{\prime}, r_{1}^{\prime}, t_{1}^{\prime}, t_{2}^{\prime}\right\}$.

Suppose that $\min I=\max \{\min$ I, min II. min III, min IV $\}$, that the best pair of functions $f$ and $g$ is from Table I. So, $\operatorname{dmin}(f, g)=\min \mathrm{I}$ $=\min \left\{r_{0}^{\prime}, r_{1}^{\prime}, t_{1}^{\prime}, t_{2}^{\prime}\right\}$ and $\alpha(X) \geq \min \left\{r_{0}^{\prime}, r_{1}^{\prime}, t_{1}^{\prime}, t_{2}^{\prime}\right\}$ where $\alpha=\sigma, \sigma_{0}, \sigma^{*}, \sigma_{0}^{*}, s$ and $s^{*}$. We will now show that $\alpha(X)=\min \left\{r_{0}^{\prime}, r_{1}^{\prime}, t_{1}^{\prime}, t_{2}^{\prime}\right\}=\operatorname{dmin}(f, g)$ where $\alpha=\sigma, \sigma_{0}, \sigma^{*}, \sigma_{0}^{*}, s$ and $s^{*}$.

Let $f^{*}, g^{*}: C \rightarrow Z$ be continuous functions from a continuum $C$ into a continuum $Z \subseteq Y$ such that $f^{*}(C) \subseteq g^{*}(C)$. We consider four subcases.

CASE 2A: $\min \mathrm{I}=r_{0}^{\prime}, r_{0}^{\prime} \leq t_{1}^{\prime}, r_{0}^{\prime} \leq r_{1}^{\prime}$, and $r_{0}^{\prime} \leq t_{2}^{\prime}$.

Observe that $\min \mathrm{II}=\min \left\{r_{1}^{\prime}, t_{2}^{\prime}, q_{4}^{\prime}\right\} \leq \min \mathrm{I}=r_{0}^{\prime}$. We can assume that $r_{0}^{\prime} \neq r_{1}^{\prime}$, since this case has been covered in case $1 \mathrm{~A}$, so $r_{0}^{\prime}<r_{1}^{\prime}$. We can assume that $r_{0}^{\prime} \neq t_{2}^{\prime}$, since this case has been covered in case $1 \mathrm{~B}$, so $r_{0}^{\prime}<t_{2}^{\prime}$. So, we assume that $\min \mathrm{II}=q_{4}^{\prime} \leq \min \mathrm{I}=r_{0}^{\prime}$. So, we have that $q_{4}^{\prime} \leq r_{0}^{\prime}=\operatorname{dmin}(f, g)$. We can assume that $q_{4}^{\prime} \neq r_{0}^{\prime}$, since this is covered in case $1 \mathrm{C}$. So, $q_{4}^{\prime}<r_{0}^{\prime}=\operatorname{dmin}(f, g)$. We define $p_{1}: Y \rightarrow \overrightarrow{Q_{1}^{\prime} Q_{1}} \cup \overrightarrow{Q_{1}^{\prime} Q_{2}}$ based on case $p_{1}, Y_{r}, 1$ for $y \in Y_{r}$ since $r_{0}^{\prime} \leq t_{1}^{\prime}$ and based on either case $p_{1}, Y_{l}, 1$ if $t_{3}^{\prime} \leq r_{2}^{\prime}$ or case $p_{1}, Y_{l}, 2$ if $r_{2}^{\prime}<t_{3}^{\prime}$ for $y \in Y_{l}$. Consider $p_{1} \circ f^{*}, p_{1} \circ g^{*}: C \rightarrow \overrightarrow{Q_{1}^{\prime} Q_{1}} \cup \overrightarrow{Q_{1}^{\prime} Q_{2}}$. Since the range is an interval there is a $c \in C$ such that $p_{1} \circ f^{*}(c)=p_{1} \circ g^{*}(c)$ and $d\left(f^{*}(c), g^{*}(c)\right) \leq \max \left\{\min \left\{t_{3}^{\prime}, r_{2}^{\prime}\right\}, q_{4}^{\prime}, r_{0}^{\prime}\right\}=r_{0}^{\prime}$.

CASE $2 \mathrm{~B}: \min \mathrm{I}=t_{1}^{\prime}, t_{1}^{\prime}<r_{0}^{\prime}, t_{1}^{\prime} \leq r_{1}^{\prime}$, and $t_{1}^{\prime} \leq t_{2}^{\prime}$.

Observe that $\min$ II $=\min \left\{r_{1}^{\prime}, t_{2}^{\prime}, q_{4}^{\prime}\right\} \leq \min \mathrm{I}=t_{1}^{\prime}$. We can assume that $t_{1}^{\prime} \neq r_{1}^{\prime}$, since this is already covered in case $1 \mathrm{~A}$, so $t_{1}^{\prime}<r_{1}^{\prime}$. We can assume that $t_{1}^{\prime} \neq t_{2}^{\prime}$, since this is already covered in case $1 \mathrm{~B}$, so $t_{1}^{\prime}<t_{2}^{\prime}$. So we assume that $\min \mathrm{II}=q_{4}^{\prime} \leq \min \mathrm{I}=t_{1}^{\prime}$. So, we have that $q_{4}^{\prime} \leq t_{1}^{\prime}=\operatorname{dmin}(f, g)$. We can assume that $q_{4}^{\prime} \neq t_{1}^{\prime}$, since this is covered in case $1 \mathrm{C}$. So, $q_{4}^{\prime}<t_{1}^{\prime}=\operatorname{dmin}(f, g)$. We define $p_{1}: Y \rightarrow \overrightarrow{Q_{1}^{\prime} Q_{1}} \cup \overrightarrow{Q_{1}^{\prime} Q_{2}}$ based on case $p_{1}, Y_{r}, 2$ for $y \in Y_{r}$ since $t_{1}^{\prime}<r_{0}^{\prime}$ and based on either case $p_{1}, Y_{l}, 1$ if $t_{3}^{\prime} \leq r_{2}^{\prime}$ or case $p_{1}, Y_{l}, 2$ if $r_{2}^{\prime}<t_{3}^{\prime}$ for $y \in Y_{l}$. Consider $p_{1} \circ f^{*}, p_{1} \circ g^{*}: C \rightarrow \overrightarrow{Q_{1}^{\prime} Q_{1}} \cup \overrightarrow{Q_{1}^{\prime} Q_{2}}$. Since the 
range is an interval there is a $c \in C$ such that $p_{1} \circ f^{*}(c)=p_{1} \circ g^{*}(c)$ and $d\left(f^{*}(c), g^{*}(c)\right) \leq \max \left\{\min \left\{t_{3}^{\prime}, r_{2}^{\prime}\right\}, q_{4}^{\prime}, t_{1}^{\prime}\right\}=t_{1}^{\prime}$.

CASE $2 \mathrm{C}: \min \mathrm{I}=r_{1}^{\prime}, r_{1}^{\prime}<r_{0}^{\prime}, r_{1}^{\prime}<t_{1}^{\prime}$, and $r_{1}^{\prime} \leq t_{2}^{\prime}$.

This case has been covered in case $1 \mathrm{~A}$.

CASE 2D: $\min \mathrm{I}=t_{2}^{\prime}, t_{2}^{\prime}<r_{0}^{\prime}, t_{2}^{\prime}<r_{1}^{\prime}$, and $t_{2}^{\prime}<t_{1}^{\prime}$.

This case has already been covered in case $1 \mathrm{~B}$.

Therefore in case 2 we conclude that $\sigma_{0}(X) \leq \operatorname{dmin}(f, g)=\min$ I. Also, $\sigma(X)=\sigma^{*}(X)$ and $\sigma_{0}(X)=\sigma_{0}^{*}(X)$ since $X$ is a simple closed curve and for all $t \in[0,1]$ there is a $t^{\prime} \in[0,1]$ such that $f(t)=g\left(t^{\prime}\right)$ and $f\left(t^{\prime}\right)=g(t)$. Consequently, $\sigma(X)=\sigma_{0}(X)=\sigma^{*}(X)=\sigma_{0}^{*}(X)=s(X)=s^{*}(X)=\min \mathrm{I}$.

The other three cases can be proved in a similar manner.

Hence, $\sigma(X)=\sigma_{0}(X)=\sigma^{*}(X)=\sigma_{0}^{*}(X)=s(X)=s^{*}(X)=$ $\max \left\{q_{j+3}^{\prime}, \min\right.$ I, min II, min III, $\left.\min \mathrm{IV}\right\}=\operatorname{fss}(X)$.

TheOREM 3.2. Let $Z$ be a continuum such that $Z \subseteq Y$, where $Y$ is the closure of the bounded component of $R^{2}-X$ and $X$ is a five star-like simple closed curve, then $\alpha(Z) \leq \operatorname{fss}(X)$, where $\alpha=\sigma, \sigma_{0}, \sigma^{*}, \sigma_{0}^{*}, s, s^{*}$.

Proof. Since each of the functions $p_{i}$ for $i=0,1,2,3,4$ were defined for any continuum $Z \subseteq Y$ where $Y$ is the closure of the bounded component of $R^{2}-X$, we see that for any such $Z, \alpha(Z) \leq \operatorname{fss}(X)$, where $\alpha=\sigma, \sigma_{0}, \sigma^{*}, \sigma_{0}^{*}, s$, and $s^{*}$.

Corollary 3.3. Let $Z$ be a simple closed curve such that $Z \subseteq Y$ where $Y$ is the closure of the bounded component of $R^{2}-X$ and $X$ is a five star-like simple closed curve, then

$$
\sigma(Z) \leq \sigma(X) .
$$

So, in this situation, the question by Howard Cook is answered in the affirmative.

Corollary 3.4. Let $Y$ be a plane separating continuum and $X$ be a five star-like simple closed curve contained in the closure of a bounded component of $R^{2}-Y$, then

$$
\sigma(X) \leq \sigma(Y) .
$$

Proof. Let $Y$ be a plane separating continuum such that $X$ is contained in one of the bounded components of $R^{2}-Y$. We can assume that the origin, $\mathcal{O}$, is in the bounded component of $R^{2}-Q$. There is an $r>0$ such that the circle with center the origin and radius $r, C(\mathcal{O}, r)$, is such that $Y$ is contained in the bounded component of $R^{2}-C(\mathcal{O}, r)$. We will show that $\sigma(Y) \geq \sigma(X)$.

We will consider the case where $\sigma(X)=\min$ II. The lines $\overleftrightarrow{Q_{2} Q_{1}}$ and $\overleftrightarrow{Q_{2} Q_{4}}$ separate the bounded component of $R^{2}-C(\mathcal{O}, r)$ into four wedges. We let $V_{0}$ be the closure of the wedge that contains $Q_{4}^{\prime}$ and $Q_{0}^{\prime}$ and $W_{0}$ be the closure 
of the wedge that is opposite $V_{0}$. The set $V_{0}-\left(\overline{Q_{4} Q_{4}^{\prime}} \cup \overline{Q_{4}^{\prime} Q_{0}} \cup \overline{Q_{0} Q_{0}^{\prime}} \cup \overline{Q_{0}^{\prime} Q_{1}}\right)$ has two components. Let $W_{0}^{\prime}$ be the closure of the component that intersects $C(\mathcal{O}, r)$. For each $z \in W_{0} \cap Y$, let $r(z) \in \overleftrightarrow{Q_{2} z} \cap W_{0} \cap C(\mathcal{O}, r)$ and for each $z \in W_{0}^{\prime} \cap Y$, let $r(z) \in \overleftrightarrow{Q_{2} z} \cap W_{0}^{\prime} \cap C(\mathcal{O}, r)$. The lines $\overleftrightarrow{Q_{4} Q_{2}}$ and $\overleftrightarrow{Q_{4} Q_{1}}$ separate the bounded component of $R^{2}-C(\mathcal{O}, r)$ into four wedges. Let $V_{1}$ be the closure of the wedge that contains $Q_{1}^{\prime}$. Let $W_{1}$ be the closure of the wedge that is opposite $V_{1}$. The set $\overline{Q_{1} Q_{1}^{\prime}} \cup \overline{Q_{1}^{\prime} Q_{2}}$ separates $V_{1}$ into two components. Let $W_{1}^{\prime}$ be the closure of the component that intersects $C(\mathcal{O}, r)$. For $z \in W_{1} \cap Y$, let $r(z) \in \overleftrightarrow{Q_{4} z} \cap W_{1} \cap C(\mathcal{O}, r)$, and for $z \in W_{1}^{\prime} \cap Y$, let $r(z) \in \overleftrightarrow{Q_{4} z} \cap W_{1}^{\prime} \cap C(\mathcal{O}, r)$. Similarly, using the lines $\overleftrightarrow{Q_{1} Q_{4}}$ and $\overleftrightarrow{Q_{1} Q_{2}}$, we define the sets $W_{2}$ and $W_{2}^{\prime}$. Note that $Q_{1} \in W_{2}$ and $Q_{2}^{\prime}, Q_{3}^{\prime} \in W_{2}^{\prime}$. We define $r$ for $z \in W_{2} \cap Y$ by $r(z) \in \overleftrightarrow{Q_{1} z} \cap W_{2} \cap C(\mathcal{O}, r)$ and for $z \in W_{2}^{\prime} \cap Y$ by $r(z) \in \overleftrightarrow{Q_{1} z} \cap W_{2}^{\prime} \cap C(\mathcal{O}, r)$. Observe that for either $x \in W_{0}$ and $y \in W_{0}^{\prime} \cap \overleftrightarrow{Q_{2} x}$ or $x \in W_{1}$ and $y \in W_{1}^{\prime} \cap \overleftrightarrow{Q_{4} x}$, or $x \in W_{2}$ and $y \in W_{2}^{\prime} \cap \overleftrightarrow{Q_{1} x}, d(x, y) \geq \min$ II.

We can rotate $X \cup Y$ in the plane about the origin so that the ray $\overrightarrow{\mathcal{O} x}$ corresponds to the positive x-axis, where $\{x\}=W_{0} \cap W_{2}^{\prime} \cap \overleftrightarrow{Q_{1} Q_{2}} \cap C(\mathcal{O}, r)$. Let $\theta_{j}$ for $j=0,1,2,3,4,5,6$, be angles in the rotated position such that $0=\theta_{0}<\theta_{1}<\theta_{2}<\theta_{3}<\theta_{4}<\theta_{5}<\theta_{6}=2 \pi$, where $r e^{i \theta_{0}}, r e^{i \theta_{3}} \in \overleftrightarrow{Q_{1} Q_{2}} \cap$ $C(\mathcal{O}, r), r e^{i \theta_{1}}, r e^{i \theta_{4}} \in \overleftrightarrow{Q_{2} Q_{4}} \cap C(\mathcal{O}, r)$, and $r e^{i \theta_{2}}, r e^{i \theta_{5}} \in \overleftrightarrow{Q_{4} Q_{1}} \cap C(\mathcal{O}, r)$. Let $f:[0,2 \pi] \rightarrow[0,2 \pi]$ be a piecewise linear, surjective function, such that $f\left(\theta_{j}\right)=j\left(\frac{\pi}{3}\right)$, and for $\theta \in\left[\theta_{j}, \theta_{j+1}\right], f(\theta)=j\left(\frac{\pi}{3}\right)+\left(\frac{\theta-\theta_{j}}{\theta_{j+1}-\theta_{j}}\right)\left(\frac{\pi}{3}\right)$ for each $j=0,1,2,3,4,5$.

Let $h: C(\mathcal{O}, r) \rightarrow C(\mathcal{O}, r)$ be the homeomorphism given by $h\left(r e^{i \theta}\right)=$ $h\left(r e^{i f(\theta)}\right)$.

We define $p: Y \rightarrow C(\mathcal{O}, r)$ by $p(y)=h \circ r(y)=h\left(r e^{i \theta y}\right)=r e^{i f(\theta y)}$ where $r(y)=r e^{i \theta y}$. We see that $p$ is an essential map from $Y$ onto $C(\mathcal{O}, r)$. Also, for each $y, z \in Y$ such that $p(y)$ and $p(z)$ are diametrically opposed to each other on $C(\mathcal{O}, r), d\left(p^{-1}\{p(y)\}, p^{-1}\{p(z)\}\right) \geq \min$ II.

So in this case, by Theorem L, $\sigma(Y) \geq \sigma(X)=\min$ II. The cases where $\sigma(X)=\min \left\{q_{i}\right\}_{i=0}^{4}$, min I, min III, or min IV can be shown in a similar manner. We conclude that in all cases $\sigma(Y) \geq \sigma(X)$.

When $\mathrm{Y}$ is a simple closed curve, the question by Howard Cook is answered in the affirmative in this situation.

\section{REFERENCES}

[1] H. Cook, W. T. Ingram and A. Lelek, A list of problems known as Houston problem book. Continua (Cincinnati, OH, 1994), 365-398, Lecture Notes in Pure and Appl. Math. 170, Dekker, New York, 1995.

[2] James F. Davis, The equivalence of zero span and zero semispan, Proc. Amer. Math. Soc. 90 (1984), 133-138. 
[3] A. Lelek, Disjoint mappings and the span of spaces, Fund. Math. 55 (1964), 199-214.

[4] A. Lelek, An example of a simple triod with surjective span smaller than span, Pacific J. Math. 64 (1976), 207-215.

[5] A. Lelek, On the surjective span and semispan of connected metric spaces, Colloq. Math 37 (1977), 35-45.

[6] K. Tkaczynska, The span and semispan of some simple closed curves, Proc. Amer. Math. Soc. 1111991.

[7] K. Tkaczynska, On the span of simple closed curves, Houston J. Math. 20 (1994), 507-528.

[8] T. West, Spans of simple closed curves, Glas. Mat. Ser. III 24(1989), 405-415.

[9] T. West, $A$ bound for the span of certain plane separating continua, Glas. Mat. Ser. III 32(1997), 291-300.

[10] T. West, Spans of certain simple closed curves and related spaces, Topology Proc. 23, Summer 1998, 363-378.

[11] T. West, Concerning the spans of certain plane separating continua, Houston J. Math. 25 (1999), 697-708.

[12] T. West, Spans of various two cells, surfaces, and simple closed curves, Glas. Mat. Ser. III 37 (2002), 383-392.

[13] T. West, Spans of continua related to indented circles, Glas. Mat. Ser. III 39 (2004), 171-183.

T. West

Department of Mathematics

University of Louisiana at Lafayette

LA 70504-1010

USA

E-mail: ThelmaRWest@Yahoo.com

Received: 23.5.2006.

Revised: 4.12.2006. 